\title{
Survey of Conference Management Systems
}

\author{
Madhur Jain \\ Jaypee Institute Of Information \\ Technology, \\ Noida,Sector -62
}

\author{
Tribhuwan K. Tewari \\ Jaypee Institute Of Information \\ Technology, \\ Noida,Sector -62
}

\author{
Sandeep K. Singh \\ Jaypee Institute Of Information \\ Technology, \\ Noida,Sector -62
}

\begin{abstract}
This article surveys various conference management systems used for managing conferences which are being held worldwide. Each system possesses some common functionality and some functionality which present that particular system. The external interaction specification techniques are further subdivided into techniques for the specification of functions, behavior, and communication. After surveying the systems, we summarize the way their functionalities are used. We have surveyed EDAS, Confious, OpenConf, ConfTool and PaperDyne under the following heads : 1)System 2) Conferences 3) TPC 4) Reviewers 5) Papers 6) Reports and 7)Notifications.
\end{abstract}

\section{General Terms}

Framework, Software, Function, Behavior, Function decomposition table, Web servers.

\section{Keywords}

Conference management System(CMS)

\section{INTRODUCTION}

Traditional conference management depends on manual operations. In the mid $90 \mathrm{~s}$, email became a reliable and costeffective way to send binary file attachments and it opened the possibility of sending papers electronically instead of by "snail" mail .However, when the number of paper submissions increases to some scale, the task of dealing with these submissions manually becomes tedious. This often results in problems such as collecting the wrong author's information, neglecting some submissions and slowing down the process of conference organization.

In the last few years, several Web-based conference management systems have been developed and used by many international conferences. They have implemented such functions as publishing conference information, handling paper submissions and reviews. These functions provide reliability and security and speed up the process of conference organization. However, this kind of Web-based system is deployed on just only one Web server, i.e., a stand-alone conference management system server in which some deficiencies exists. The deficiencies provide a challenge to design and develop a new system on the current system which eliminates those existing deficiencies.

The paper is divided into four sections Section II describes finding key features of the designed framework for comparisons along with their description section III Compares the conference management systems according to the system features identified followed by Section IV for conclusion which discuss the need for a system which uses multiple web servers.

\section{FRAMEWORK FOR COMPARISONS}

The basic concept in the framework of comparison is that of a system. A system is an assembly of parts forming a complex or unitary whole that serves a useful purpose. In wide sense, organizations, pieces of furniture, computers, cars, and softwares are examples of systems. It is crucial that the parts of a system interact with each other efficiently and in sync in such a way that they cause the system as a whole to have a useful function for one or more entities in the environment of the system.

In order for a collection of interacting items to count as a system, the interactions among the items must produce a coherent and useful overall behavior.

Interactions can always be partitioned into pieces that are useful for the environment, called functions.

Second, system interactions are by nature always communications with one or more entities in the environment of the system.

Third, the way in which functions are ordered in time is called the behavior of the system. Functions, communications, and behavior are system properties. There are other kinds of system properties, such as security and user-friendliness, but these are not specified by the techniques reviewed in this survey and we leave these out of consideration.

If we add information related to functions and components in a table and indicate in each entry what the function is that the component should have in order to play this role in the system function, then we call the resulting table a function decomposition table.

The following list relates these to the function decomposition table of the system.

- System functions. These are the useful pieces of behavior offered to the environment of the system, and correspond to items in the top row of the table.

- System behavior. This is the behavior of the system over time. This concern the way in which the functions in the top row are, can, or must be ordered in time. It thus concerns the entire top row.

- System communication. This concerns the communication of the system with external entities in its environment. It does not correspond to an aspect of the function decomposition table of the system, but it would correspond to a column of the function decomposition table of the environment, of which the system is one component.

- Conceptual decomposition. These are the conceptual components of the system, defined in terms of their meaning for 
the external environment, and correspond to the items in the leftmost column of the table.

Component functions. These are the useful pieces of behavior offered by the components to their environment, and correspond to entries in the table.

Component behavior. This is the way in which component functions are, can, or must be ordered in time, and corresponds with a row in the function decomposition table. This is a way in which the components interact in order to realize the external functions, and corresponds to the columns of the function decomposition table. In each column, the component functions are listed that interact to realize the external function.

- Component communication. This is the way in which the components interact in order to realize the external functions, and corresponds to the columns of the function decomposition table. In each column, the component functions are listed that interact to realize the external function.

Key features and their description is given below:

\subsection{System Function Comparison}

Under the system category the following functions were compared Registering to the system, Creating profile, Editing user profile, Help, Conference invoice, Backup database, Multiple account management, Printing problem management, Plagiarism check, Page charges, Registering to the system, profile creation and database driven service is present in all the system. EDAS and OPENCONF enables user to edit his profile and provides online help. EDAS provides the feature of Conference Invoice, multiple account management. EDAS charges for extra pages, Printing problem management is notified using emails.EDAS uses DOCOLOC for plagiarism check. Confious, OpenConf and ConfTool provide the feature to backup database.

\subsection{Conference Function Comparison}

The basic conference related functions are Creating conferences, setting up dates and deadlines, setting up the maximum number of reviewers per paper in a conference, Specifying topics for conference and reviews, Defining roles like Chairs, Authors, Reviewers and TPC. Post conference creation, the functions are Registering to a particular conference which has not ended, Listing the conferences where the user is a chair, Viewing author permissions, Conflict of interest, Assigning number for review purpose, Scheduling of the conference, Multi conference support, Travel Grants, Copyright management, Phase management, Moving papers among tracks, Sub conference creation, Registering yourself for dinner, Conference logo, View all attendees and Printing badges.Features like Conference creation, Defining roles like authors, reviewers, chairs and Technical Program Committee Registering yourself to conference, Conflict of interest, Listing all the conference where user is chair, Multi conference support(Allowing more than one conference) are provided by all the systems.

Each conference must have at least one chair. Only chairs can add TPC members, change the conference configuration, add review forms and templates and perform other administrative duties. Chairs can submit papers at any time, even after track deadlines have passed.

Chairs are allowed to author papers, but if they are looking at their own papers, their privileges are reduced to that of normal authors. Thus, they cannot see reviewer names or make review assignments for these papers.

EDAS, Confious, OpenConf, ConfTool provides the feature for Setting up dates and deadlines, specifying topics for conference and reviews.

EDAS alone provides features like Moving papers among tracks, Sub conference creation, Registering yourself for dinner, Conference logo i.e. uploading the logo of your conference, View all attendees, Printing badges for the conference and provision of travel grants.

In EDAS, Papers can be submitted only to conferences that have one or more tracks. Each track can have its own paper registration and submission deadlines, acceptable paper formats (PDF, PowerPoint, etc.), maximum page count and margins, but all tracks within a conference share the same TPC, chairs and letters. TPC members can be assigned to a

specific track, however. However, tracks within a conference share the same accept and reject notices, but each track can define a block of text for accepted and rejected papers that can be included in the author notification. Track information can also be used for creating a conference program.Each such sub-conference can have its own TPC, chairs, letters.

EDAS, Confious, ConfTool has the feature of copyright management.

The feature of phase management which comprises of nine phases viz. Setup Phase, Invitation Phase, Abstract Submission Phase, Bidding Phase, Full paper submission phase, Assignment Phase, Reviewing Phase, Decision Phase and Publish Reviewing Results phase is provided by Confious, OpenConf and ConfTool.

Assigning papers for the purpose of review can be done in two ways, automatically in which the system assigns the papers to reviewers and the other way is manually. EDAS, ConfTool, Confious allows both automatic and manual paper assignment.

\subsection{Technical Program Committee}

A TPC member can be assigned TPC reviews and may have special privileges to see reviews and to assign regular reviews to papers, depending on the conference configuration. TPC members can be assigned to TPC groups and can register for the TPC meeting. TPC members can be assigned to a specific track and then only see papers for that track. Review questions can be designated as being viewable by role, e.g., only by a chair, group leader or TPC member. All types of TPC members are only part of the automatic paper assignment if they are flagged as reviewing papers. That is the default for reviewers and TPC members, but not for group leaders and chairs. TPC members can be grouped into TPC groups, with a group leader. TPC groups are useful for large TPC meetings; they are not, however, used for assigning papers. Papers can be assigned to TPC groups, so that members of the group can see the reviews. Functions under comparison which are related to Technical Program Committee here after referred to as TPC are as follows- Creation of TPCs, formation of TPC groups, Viewing TPC stats for reviews and registering a TPC meeting. In the survey it was found out that only EDAS provides functionalities like creation of TPC, formation of TPC groups, viewing TPC stats for reviews and registering a TPC meeting, while Confious allows the creation of TPCs only. 


\subsection{Papers}

Submitting a paper to a already registered and valid conference, Viewing all the papers submitted by a user including accepted, rejected, published and withdrawn, viewing details of submitted paper, maintaining a log: Identify all the actions taken on the paper, Re-uploading a previously uploaded paper incase some changes are made, Downloading papers by registered users, checking the paper format.The basic features of submitting a paper to an already registered and valid conference, re-uploading a previously submitted paper in which some changes are made to a valid conference, allowing registered users to download papers, viewing paper details are provided by all the products considered under this survey i.e. by EDAS, Confious, OpenConf, ConfTool and Paperdyne.Paper log which contains all the information regarding what all phases has the paper undergone is given by EDAS, Confious. Only EDAS has the feature of checking the paper format. It checks whether the submitted paper is in two column format or not. PDF files can be checked to make sure that font sizes, margins and page counts follow conference rules. The system can check for missing fonts and ensure proper PDF structure, according to IEEE PDF rules. Paper data can be exported into Excel-compatible files, with a selectable set of fields. Accepted papers can be output as BibTeX records.

\subsection{Reviewers}

Review assignments from conferences that have not ended are shown, Review Form, Edit Review, laim(Request)paper to review, Review Papers. There are two kinds of review forms: for regular reviews and TPC reviews. Both types of reviews can be done by members of the TPC or by external persons not part of the technical program committee. In case of a one-level review system, where all reviews are the same, it does not much matter which one you use, as long as you assign reviewers or TPC members consistently to either the 'review' or 'TPC review' category.

EDAS, Confious, OpenConf, ConfTool and Paperdyne allows review assignments for all the conferences. Three-tier review model, consisting of one or more conference chairs, TPC members and reviewers. EDAS, Confious, OpenConf support two tier review process which comprises of technical program committee plus external reviewers.

Confious and ConfTool provides a dynamic review form whereas EDAS provides a static review form. Editing of reviews is also provided by EDAS, Confious and OpenConf.

- Component communication. This is the way in which the components interact in order to realize the external functions, and corresponds to the columns of the function decomposition table. In each column, the component functions are listed that interact to realize the external function.

\subsection{Notifications}

Send conference related mails to registered users, Communicate within a particular group, Reminders are the features compared under the head of notification EDAS, Confious, ConfTool and OpenConf is capable of sending reminders during the various phases of a paper.
Both EDAS and Confious provides a dynamic format and templates for sending conference related mails to registered users. Communication within a group is also provided.

\subsection{Reports}

Conference Information Reports, Decision Driven Reports and Assignment Driven Reports are specifically provided by Confious in a separate section.

\subsection{Assigning Reviewers and TPC Members}

\subsubsection{Automatically}

TPC members are sent an email containing instructions and a link. From the link, TPC members can then claim a list of papers. There is no inherent deadline, but obviously claims submitted after the assignment is run are not taken into account. The list shown to TPC members is limited to papers that match at least one topic of interest. It excludes papers where there is a known conflict of interest. If TPC members are assigned to specific tracks, only papers in their track are shown. TPC members can assign each paper into four categories, namely papers that they would like to review, can review, are willing to review if absolutely needed and cannot review. Reviewers are only assigned papers from the first three categories.

The goal of the automatic review assignment is to ensure that every paper has the required number of TPC and regular reviews. A secondary objective is to ensure that papers are reviewed by TPC members that are most interested in the paper.

After those criteria, the algorithm tries to ensure that the review load is balanced between reviewers. Clearly, it's impossible to optimize for all three criteria at once, so some amount of unequal distribution is unavoidable.

\subsubsection{Manually}

After defining a review form, EDAS allows you to add reviewers. The TPC member or reviewer will receive an email asking him to review the paper. The TPC member or reviewer can accept or decline this invitation. Once he has accepted, he will be sent another email containing detailed review instructions. Reviewers and TPC members can always check their EDAS home page to find the papers that they have been assigned. Reviewers can receive an email notification for each review they have been assigned, or a single email if one or more new reviews have been assigned.

A TPC reviewer, if allowed by the review configuration, can assign additional non-TPC reviews for a paper, but no additional TPC reviews. Thus, there's one additional review outstanding after the TPC member assigns another review. If allowed by configuration, both a TPC member and a reviewer can delegate ("hand off") their review to another person. They remain listed as the original reviewer for the paper and can check the status of the review and view the review itself once it is done. 


\section{COMPARISON BASED ON SYSTEM FEATURES}

The comparison of the surveyed management systems based on the system features can be tabulated and summarized as[1]-[12]

Table 1:Comparison of the different CMS based on System Features

\begin{tabular}{|c|c|c|c|c|c|c|}
\hline S.no & System & EDAS & Confious & OpenConf & ConfTool & PaperDyne \\
\hline 1 & Register to system & Yes & Yes & Yes & Yes & Yes \\
\hline 2 & Create Profile & Yes & Yes & Yes & Yes & Yes \\
\hline 3 & Create Conference & Yes & Yes & Yes & Yes & Yes \\
\hline 4 & $\begin{array}{l}\text { Dates and deadlines: Displays the conference } \\
\text { related dates and deadlines }\end{array}$ & Yes & Yes & Yes & N.A & N.A \\
\hline 5 & $\begin{array}{l}\text { Set max. no. of reviewers per paper in a } \\
\text { conference }\end{array}$ & Yes & Yes & N.A & N.A & N.A \\
\hline 6 & Specify topics for conference and reviews & Yes & Yes & Yes & Yes & N.A \\
\hline 7 & Define Roles-Chair, Authors, Reviewers, TPC & Yes & Yes & Yes & Yes & Yes \\
\hline 8 & $\begin{array}{l}\text { Profile: Change your account, show your } \\
\text { profile, change your password, upload a photo, } \\
\text { update your areas of interest or update your } \\
\text { conflicts of interest }\end{array}$ & Yes & N.A & N.A & N.A & N.A \\
\hline 9 & $\begin{array}{l}\text { Register :- Register yourself to a conference } \\
\text { that has not ended. }\end{array}$ & Yes & Yes & Yes & Yes & Yes \\
\hline 10 & $\begin{array}{l}\text { Submit Paper to a already registered and valid } \\
\text { conference. }\end{array}$ & Yes & Yes & Yes & Yes & Yes \\
\hline 11 & $\begin{array}{l}\text { Reviews: review assignments from conferences } \\
\text { that have not ended are shown }\end{array}$ & Yes & Yes & Yes & Yes & Yes \\
\hline 12 & $\begin{array}{l}\text { Chairing:-Lists the conferences where the user } \\
\text { is a chair }\end{array}$ & Yes & Yes & Yes & Yes & Yes \\
\hline 13 & $\begin{array}{l}\text { Author permissions: Shows, Assign } \\
\text { Permissions for author. }\end{array}$ & Yes & Yes & N.A & N.A & N.A \\
\hline 14 & Technical Program Committee (TPC) & Yes & Yes & N.A & N.A & N.A \\
\hline
\end{tabular}




\begin{tabular}{|c|c|c|c|c|c|c|}
\hline 15 & $\begin{array}{l}\text { View all the papers submitted by the user. } \\
\text { Including Accepted, Rejected, Published and } \\
\text { Withdrawn }\end{array}$ & Yes & N.A & N.A & N.A & N.A \\
\hline 16 & View details of submitted paper & Yes & Yes & N.A & Yes & N.A \\
\hline 17 & Log :Identify all the actions taken on the paper & Yes & Yes & N.A & N.A & N.A \\
\hline 18 & $\begin{array}{l}\text { Review - Three-tier review model, consisting of } \\
\text { one or more conference chairs, TPC members } \\
\text { and reviewers }\end{array}$ & Yes & Two Tier & Two Tier & N.A & N.A \\
\hline 19 & Conflict Of Interest & Yes & Yes & Yes & Yes & Yes \\
\hline 20 & Paper Assignment - For review purpose & Auto/Manual & Auto/Manual & N.A & $\begin{array}{c}\text { Auto/Manua } \\
1\end{array}$ & N.A \\
\hline 21 & Review Form & Static & Dynamic & N.A & Dynamic & N.A \\
\hline 22 & $\begin{array}{l}\text { Reupload a previously uploaded paper incase } \\
\text { some changes are made. }\end{array}$ & Yes & Yes & N.A & Yes & N.A \\
\hline 23 & Registered users can download papers & Yes & Yes & Yes & Yes & Yes \\
\hline 24 & Schedule of the conference & Yes & Yes & N.A & N.A & N.A \\
\hline 25 & Help & Yes & No & N.A & N.A & N.A \\
\hline 26 & $\begin{array}{l}\text { Multiconference support:Allow more than once } \\
\text { conference to be handled by the system. }\end{array}$ & Yes & Yes & Yes & Yes & Yes \\
\hline 27 & Transaction based operations (database driven) & Yes & Yes & Yes & Yes & Yes \\
\hline 28 & $\begin{array}{l}\text { Send conference related mails to registered } \\
\text { users }\end{array}$ & Yes & $\begin{array}{l}\text { Yes(Dynamic } \\
\text { format) }\end{array}$ & N.A & N.A & N.A \\
\hline 29 & Communicate within a particular group. & No & Yes & N.A & N.A & N.A \\
\hline 30 & Conference-Information Reports - & No & Yes & N.A & N.A & N.A \\
\hline 31 & Decision-Driven Reports & No & Yes & N.A & N.A & N.A \\
\hline 32 & Assignment-Driven Reports & No & Yes & N.A & N.A & N.A \\
\hline
\end{tabular}




\begin{tabular}{|c|c|c|c|c|c|c|}
\hline 33 & Travel Grants & Yes & N.A & N.A & N.A & N.A \\
\hline 34 & Conference Invoice(Bill) & Yes & N.A & N.A & N.A & N.A \\
\hline 35 & Copyright management & Yes & Yes & N.A & Yes & N.A \\
\hline 36 & Paper Format & $\begin{array}{c}\text { Two column } \\
\text { check }\end{array}$ & N.A & N.A & N.A & N.A \\
\hline 37 & Phase Management(9 phases) & N.A & Yes & Yes & Yes & N.A \\
\hline 38 & Backup Database & N.A & Yes & N.A & Yes & N.A \\
\hline 39 & Multiple Account Management & Yes & No & No & No & No \\
\hline 40 & Printing Problem Management & $\begin{array}{l}\text { Notification } \\
\text { through mail }\end{array}$ & N.A. & N.A. & N.A. & N.A \\
\hline 41 & Plagiarism Check & Docoloc & N.A. & N.A. & N.A. & N.A \\
\hline 42 & Page Charges & Extra Pages & N.A. & N.A. & N.A. & N.A \\
\hline 43 & Move papers among tracks & Yes & N.A. & N.A. & N.A. & N.A \\
\hline 44 & Sub Conference & Yes & No & No & No & No \\
\hline 45 & Edit Review & Yes & Yes & N.A. & Yes & N.A \\
\hline 46 & Claim(Request)paper to review & Yes & Yes & N.A. & N.A. & N.A \\
\hline 47 & Review Papers & Yes & Yes & N.A. & N.A. & N.A \\
\hline 48 & Form TPC groups & Yes & No & N.A. & N.A. & N.A \\
\hline 49 & View TPC stats for reviews & Yes & No & N.A. & N.A. & N.A \\
\hline 50 & Register TPC meeting & Yes & No & N.A. & N.A. & N.A \\
\hline 51 & Register Dinner Choice & Yes & No & N.A. & N.A. & N.A \\
\hline 52 & Conference logo & Yes & No & N.A. & N.A. & N.A \\
\hline 53 & View all attendees & Yes & No & N.A. & N.A. & N.A \\
\hline 54 & Print name badges & Yes & No & N.A. & N.A. & N.A \\
\hline 55 & Reminders & Yes & Yes & Yes & Yes & N.A \\
\hline
\end{tabular}

\section{CONCLUSION}

Based on the survey carried out, we found that EDAS is the richest in terms of features and most popular among the academic and scientific community. According to the data provided by EDAS for June'06, [8] it has roughly managed 70,000 papers across 660 conferences. Besides this there are over 100,000 reviewers and authors registered to the EDAS system. Aimed at the mentioned practical problems faced, we propose to design a conference management system where not only the current shortcomings are taken care of but also includes some new features like live audio - video conferencing and artificially intelligent software package to act as conference manager.

The conference can be seen as various phases viz. paper submission phase, reviewing phase etc. These phases can be managed by the system itself once various deadlines are set. The program committee chair can of course override the system anytime and handle everything manually.

It is quite possible for someone to submit a plagiarized work. The system can employ some technique to detect the plagiarism and report the same. 
Papers in a program are required to be submitted in a particular format, which is different for every program. The system shall check the format of the paper by the rules defined by the program committee and intimate its correctness to the author of the paper. The proposed system also incorporates a paper format builder where author needs only to feed in the text and the system can print the document in the format asked by the author.

The system also includes various discussion boards where the paper reviewers, program committee members, chair and authors can interact at various levels.

The system shall also include live audio and video conferencing so as to enable interaction with the people unable to be present themselves at the venue. It would include a small, optionally downloadable software package too, which could be installed on the system at the venue of the program and it would simply act as a replacement for the session manager.

When provided with an internet connection, speakers and a microphone, it would do everything from welcoming the guests and inviting the speakers till closing of the session according to the timings set by the chair and considering the conditions at the live program.

\section{REFERENCES}

[1] Timothy K. Shih, Jiung-Yao Huang, Jason C. Hung, and TeHua Wang, Wen-Chang Pai“The design and implementation of a virtual conference system ", COMPSAC 2000.
[2] Cheng Zheng, Weiming Shen, Qinghua Zheng, Feng Tian, "Design and implementation of a collaborative conference management system". CSCWD 2008.

[3] Yordan Kalmukov "Analysis and experimental study of an algorithm for automatic assignment of reviewers to papersInternational Conference on Computer Systems and Technologies “. CompSysTech'07

[4] Timothy K. Shih, Jason C. Hung, Te-Hua Wang, Yu-Shian Chen and Sheng-En Yeh "Virtual Conference Management System"ICOIN 2001

[5] Zhengwei Gu, Xin Jin, Bipin C. Desai "CONFSYS: The CINDI Conference Support System” IDEAS 2003

[6] Zhang Qian, Liu Shi-jun, Meng Xiang-xu "The Research and Implementation of Turning Conference Management System into a Service"APSCC 2007

[7] EDAS, available at ehttp://edas.info

[8] Confious - The Conference Nous, available at http://www.confious.com

[9] ConfTool Management Tool, available at http://www.conftool.net

[10] Paperdyn The Conference Management System, available at http://paperdyne.com.

[11] OpenConf Peer Review \& Conference Management Made Simple, available at http://openconf.org. 